\title{
Evaluation of sequential organ failure assessment (SOFA) score efficiency in predicting the mortality of intensive care unit admitted COVID-19 patients
}

\author{
Mohammad Haghighi ${ }^{1}$, Hossein Khoshrang ${ }^{1}$, Siamak Rimaz', Tofigh Yaghubi Kalurazi², Zahra Atrkar \\ Roushan ${ }^{3}$, Samaneh Ghazanfar Tehran 1,*, Paniz Rezaei ${ }^{1}$, Niloofar Faraji4 \\ ${ }^{1}$ Anesthesiology Research Center, Department of Anesthesiology, Alzahra Hospital, Guilan University of Medical \\ Sciences, Rasht, Iran \\ ${ }^{2}$ Department of Health, Nutrition and Infectious Disease, School of Medicine, Guilan University of Medical Sciences, \\ Rasht, Iran \\ 3Department of Biostatistics, Faculty of Medicine, Guilan University of Medical Sciences, Rasht, Iran \\ 4Razi Clinical Research Development Unit, Razi Hospital, Guilan University of Medical Sciences, Rasht, Iran
}

\begin{abstract}
Assessing the severity of the disease at the time of hospitalization can reduce the mortality of patients with coronavirus disease 2019 (COVID-19). For this stance, various scoring systems have been described to predict mortality rates. Sequential organ failure assessment (SOFA) is one of the scoring systems which have been used in this study. In order to calculate the SOFA score, demographic and characteristics information, clinical status, and laboratory findings were recorded from 154 patients with COVID-19, who have been admitted to the intensive care unit (ICU) for a period of 6 months. SOFA score was calculated in three time periods at the time of hospitalization, 72 hours after hospitalization, and the last day of hospitalization. Based on the outcome of the disease (death or recovery), patients were divided into two groups, and the results were analyzed in both groups. Statistical analysis has represented that the SOFA score was significantly higher in patients who died to compare with recovered ones in all time periods. Our findings suggest that SOFA scoring system can be used to predict mortality rate in ICU admitted COVID-19 patients.
\end{abstract}

Keywords: COVID-19, SOFA score, ICU, Mortality

\section{Introduction}

In December 2019, several cases of pneumonia with unknown etiology were reported in Wuhan City, Hubei Province, China. In the early stages of pneumonia, symptoms of acute respiratory infection occurred, and some patients progressed rapidly to acute respiratory failure and other serious complications [1]. On February 11, 2020, the World Health Organization (WHO) officially named the new coronavirus infection coronavirus disease 2019 (COVID-19), and the International Committee of the Red Cross study group named it as severe acute respiratory syndrome coronavirus 2 (SARS-CoV-2) [2]. Four months since the advent of COVID-19 in China, the disease has spread rapidly to other parts of the world and has become an international threat [3]. The mortality rate in patients with COVID-19 with severe conditions has been reported to be fluctuated

\section{"Corresponding author:}

Samaneh Ghazanfar Tehran, MD

Anesthesiology Research Center, Department of Anesthesiology, Alzahra Hospital, Guilan University of Medical Sciences, Rasht, Iran

Tel/Fax: +98911 2318819

Email: tehranisamaneh88rasht@gmail.com

(C) The Author(s) 2021

http://orcid.org/oooo-0002-9910-2394

Received: August, 21, 2021

Accepted: September, 21, 2021
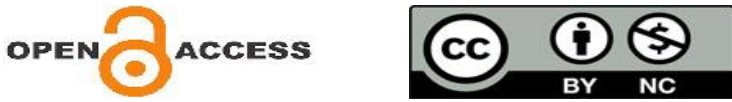
between $11 \%$ and $61 \%$ [1, 4-6]. Various studies have shown that COVID-19 affects different organs and its complications include pneumonia, liver and kidney disorders, heart damage, immunodeficiency, and coagulation disorders, which are in the result of cell and organotropism [7, 8]. Sepsis is a life-threatening complication of infection and occurs in the form of physiological, pathological, and biochemical disorders $[9,10]$.

One of the key strategies to improve the treatment of sepsis is to prioritize patients in terms of the risk of adverse outcomes. This can help physicians to prioritize initial treatments such as antibiotics and fluid therapy [11]. In critically ill patients, early medical interventions to reduce mortality depend on effective early assessment methods. A scoring system could be helpful to determine the prognosis and accelerate practical evaluation by physicians to identify these patients and could be useful in providing information to relatives of patients, treatment decisions, and guidance in allocating resources [12]. However, there is currently no specific scoring system for evaluating patients with COVID-19. The organ failure assessment score (SOFA) system can be used to determine prognosis and predict mortality. SOFA was first described in 1996, and it was used as a tool to evaluate organ failure in sepsis and also to evaluate the effects of treatments such as mechanical ventilation and vasopressor on organ dysfunction [13]. SOFA describes dysfunction of various organs through several parameters, including oxygenation index (ratio of arterial oxygen pressure to the percentage of inhaled oxygen), mean arterial pressure, Glasgow Coma Scale, creatinine or urine volume, bilirubin, and platelets, respectively for the respiratory system, circulation, neurology, renal, hepatic and coagulation. Each of these parameters is assigned o to 4 points, and the sum of these points is o to 24, which determines the overall SOFA score. The higher score indicates the worsening of the organ disorder. Numerous studies have shown that SOFA can predict morbidity and mortality and has been used for evaluation in critically ill patients and in the intensive care unit (ICU) [12, 14-16].

This study has been conducted since there is no special scoring system in patients with COVID-19, and on the other hand, there is no performed research on SOFA score in patients with COVID-19, who have been admitted to the ICU in Iran. This present study to investigate the clinical characteristics, medical records and laboratory results of ICU admitted COVID-19 patients to further evaluation for the role of SOFA score in predicting the complexity of the disease in three different time periods and help to identify the related factors.

\section{Materials and Methods}

\subsection{Study design}

This present retrospective analytical crosssectional study was performed on patients with COVID-19, who have been admitted to the ICU of Razi Hospital in Rasht, Iran, after receiving the approval of the Vice-Chancellor for Research and the code of ethics number IR.GUMS.REC.1399.509. Inclusion criteria included all patients with a definitive diagnosis of COVID-19 based on a positive nasopharyngeal PCR test, hospitalization in the ICU of Razi Hospital in Rasht, Iran, from April 1 to September 22, 2020. Exclusion criteria included patients with insufficient information about their condition, and also patients who had been admitted to the ICU before April 1, 2020 or were still admitted to the ICU after September 22, 2020. In this regard, the checklist has been used with the following items: age, sex, underlying disease history, clinical status, and laboratory results, which have been documented in the medical records of ICU, admitted COVID-19 patients, to calculate the SOFA score. The SOFA score of patients in three time periods was calculated at the time of admission to the ICU, 72 hours after admission, and the last day of hospitalization in the ICU. Based on the outcome of the disease (death or discharge), patients were divided into two groups.

\subsection{Data analysis}

Data analysis was done through SPSS software version 22 (SPSS Inc., USA). For variables with normal distribution, descriptive analysis was performed using mean and standard deviation. Number and percentage were used to report qualitative variables. Chi-square test, independent ttest, logistic regression, and Mann Whitney test were used to evaluate the study's objectives.

\section{Results}

Demographic information, including age, sex, and comorbidities in the two groups of discharged and deceased patients, is given in Table 1. In general, the mean age of the patients was $60.07 \pm 15.73$ years, with 
$53.2 \%$ of males. The Chi-square test has shown no statistically significant relationship between gender and deceased or recovered patients with COVID-19 disease $(\mathrm{P}=0.54)$. However, there was a remarkable relationship between the two groups in terms of age, so that the mean age in the deceased patients was higher than the other group $(\mathrm{P}=0.002)$. Also, there was a noteworthy relationship between the presence of underlying disease in patients admitted to ICU and the outcome of treatment, but there was no difference in the distribution of underlying disease in hospitalized patients $(\mathrm{P}>0.05)$.

SOFA score analysis with Mann Whitney U test has represented a significant difference between SOFA score values in the three time periods in the two groups (Table 1). Logistic regression on SOFA score at the time of admission in the ICU as an intervening variable in predicting patient mortality has illustrated that increasing each SOFA score at the time of hospitalization can increase the probability of mortality by 1.27 times $(\mathrm{P}<0.001)$. Also, logistic regression on SOFA score at 72 hours after admission in the ICU as an intervening variable in predicting patient mortality has illustrated that increasing each SOFA score at 72 hours after admission can increase the probability of mortality by 1.47 times $(\mathrm{P}<0.001)$. Logistic regression on SOFA score on the last day of hospitalization in the ICU as an intervening variable in predicting patient mortality has illustrated that increasing each SOFA score on the previous day of hospitalization could increase the probability of mortality by 1.9 times $(\mathrm{P}<0.001)$, Table 2. Mann Whitney U test has illustrated that there was a significant difference between the values of SOFA score at the time of admission, 72 hours after admission, and the last day of admission according to the presence of underlying diseases in the studied patients $(\mathrm{P}=0.02, \mathrm{P}=0.007, \mathrm{P}=0.01)$. Among the underlying diseases, there was a significant difference between the values of SOFA scores in the studied periods only in heart, kidney, and liver diseases. SOFA score in heart disease at 72 hours postoperatively, in kidney disease in all stages, and in liver disease in the first and last day of hospitalization was significantly higher than the other group (Table 3).

\section{Discussion}

The COVID-19 disease can easily lead to acute respiratory distress, multiorgan dysfunction, acute heart damage, acute kidney damage, and even death in the case of severity [17]. During the corona pandemic, the increase in the number of critically ill patients and the limited medical resources leads to global concern worldwide [18, 19]. Therefore, the initial evaluation of patients with severe COVID-19 disease is crucial for monitoring and early medical interventions. Consequently, the important duty of physicians is to screen patients with a high mortality rate among very critically ill patients [20]. A scoring system can help physicians to be quick and accurate diagnosis to make treatment decisions. However, a simple and effective way to assess the severity and outcome of patients with COVID-19 is challenging for physicians [17]. The SOFA score is one of the scoring systems that is used to assess organ failure and can predict the severity and outcome of the disease. For this purpose, the SOFA scale is used in the health care system, which has high accuracy, but on the other hand, it's complicated and time-consuming because it requires the evaluation of several parameters [17, 21]. A study has reported that modified sequential organ failure assessment (MSOFA) score also can be useful as well as the SOFA and it's easier to implement in resource-constrained settings [22]. Organ dysfunction is associated with a high mortality rate and a large percentage of the budget in the ICU. The SOFA score can be used to assess organ dysfunction or failure and morbidity. However, this system was first developed to describe organ function rather than predict patient outcomes. Studies have shown a remarkable association between organ function discrepancy and mortality [23].

Since there is no special scoring system for risk assessment in patients with COVID-19, we conducted this study to evaluate the effectiveness of the SOFA score in critically ill patients to identify an effective risk scoring system in COVID-19 patients. This present study has represented that the SOFA score was higher in patients who died to compare with recovered ones in all time periods. Also, based on logistic regression, the SOFA score was identified as a predictor of mortality in critically ill patients in the ICU. 
Table 1. Demographic, clinical findings and SOFA values of hospitalized patients by treatment outcome

\begin{tabular}{|c|c|c|c|c|}
\hline Variables & & Death & Recovery & $P$ value \\
\hline \multirow{2}{*}{ Gender } & Male & $45(54.9)$ & $37(45.1)$ & \multirow{2}{*}{0.54} \\
\hline & Female & $43(59.7)$ & $29(40.3)$ & \\
\hline Age $($ Mean \pm SD) & & $63.45 \pm 14.78$ & $55.57 \pm 15.94$ & 0.002 \\
\hline \multirow{2}{*}{ Underlying disease } & Yes & $73(62.4)$ & 44 (37.6) & \multirow{2}{*}{0.019} \\
\hline & No & $15(40.5)$ & $22(59.5)$ & \\
\hline \multirow{9}{*}{ Underlying disease } & Diabetes & $24(47.1)$ & $27(52.9)$ & 0.49 \\
\hline & High blood pressure & $22(34.4)$ & $42(65.6)$ & 0.07 \\
\hline & Heart disease & $16(36.4)$ & $28(63.6)$ & 0.30 \\
\hline & Respiratory disease & $8(36.4)$ & $14(63.6)$ & 0.50 \\
\hline & Kidney disease & $5(33.3)$ & $10(67.7)$ & 0.43 \\
\hline & Liver disease & - & $3(100)$ & 0.26 \\
\hline & Neurological disease & $4(36.4)$ & $7(63.6)$ & 0.65 \\
\hline & Malignancy & $6(42.9)$ & $8(57.1)$ & 1.00 \\
\hline & Rheumatic disease & $3(60)$ & $2(40)$ & 0.65 \\
\hline \multirow{3}{*}{ SOFA score } & At the time of admission & $8.76 \pm 3.69$ & $6.12 \pm 2.87$ & 0.0001 \\
\hline & 72 hours after admission & $9.92 \pm 3.9$ & $5.6 \pm 2.76$ & 0.0001 \\
\hline & Last day of admission & $11.07 \pm 3.92$ & $4.65 \pm 2.09$ & 0.0001 \\
\hline
\end{tabular}

Table 2. Comparison of SOFA Score predictive power by measurement times

\begin{tabular}{lcccc}
\hline Variables & Time of admission & Incidence rate (OR) & Confidence interval (CI) & $\boldsymbol{P}$ value \\
\hline \multirow{3}{*}{ SOFA score } & At the time of admission & 1.27 & $1.13-1.42$ & $<0.001$ \\
\cline { 2 - 5 } & 72 hours after admission & 1.47 & $1.27-1.70$ & $<0.001$ \\
\cline { 2 - 6 } & Last day of admission & 1.90 & $1.55-2.34$ & $<0.001$
\end{tabular}

A study by Liu et al. [24] has evaluated the efficacy of SOFA compared to quick SOFA (qSOFA) Score, which identifies patients outside the ICU with suspected infection that are at a high risk for inhospital mortality and is composed of 3 variables (respiratory rate, systolic blood pressure, and altered mental status), in predicting the outcome of patients with COVID-19, $\mathrm{SOFA} \geq 3$, and $\mathrm{qSOFA} \geq 1$ are related to mortality in severe COVID-19 disease. In addition, SOFA is a very sensitive marker in diagnosing inhospital mortality and is preferred to qSOFA for predicting outcomes [21]. Another study by Raith et al., which has been conducted to evaluate the power of SOFA, qSOFA, and Systemic Inflammatory Response Syndrome (SIRS) criteria, which assessment includes 4 clinical variables (temperature, heart rate, respiratory rate, and white blood cell count), in predicting the outcome of patients suspected of infection admitted to the ICU, has reported that increasing SOFA score is more than twice as strong predictor for mortality in comparison to qSOFA and SIRS criteria [10]. Yang et al. by considering 60-day mortality, have reported the patients with a higher SOFA score $(5 \leq)$ had a higher risk of mortality than patients with a lower SOFA score ( $5 \geq$ ), which is similar to our study [17]. Also, in the study of Chen et al., SOFA score, along with old age and high underlying disease, were identified as two independent risk factors for death in patients with COVID-19 [12]. However, a study by Raschke et al., which examined the accuracy of the SOFA score to determine the possible mortality of patients with COVID-19 pneumonia requiring mechanical ventilation, showed that the power of SOFA to predict mortality in patients with COVID-19 pneumonia was weak and significantly less valuable than the age factor [25]; they justified the results by referring to the previous studies, which stated SOFA score in 3 subgroups (pulmonary, hepatic and renal disease) and the relation with mortality in COVID-19 patients $[25,26]$. Our study showed that there was a statistically significant relationship between SOFA score and underlying disease at baseline, 72 hours after hospitalization, and the last day of hospitalization. 
Table 3. Comparison of SOFA Score scores in the studied time periods according to the presence or absence of underlying disease in hospitalized patients

\begin{tabular}{|c|c|c|c|c|}
\hline Variables & SOFA Score & Underlying disease & Mean \pm SD & P value \\
\hline \multirow{6}{*}{ Underlying disease } & \multirow{2}{*}{ At the time of admission } & Yes & $7.95 \pm 3.58$ & \multirow{2}{*}{0.02} \\
\hline & & No & $6.59 \pm 3.53$ & \\
\hline & \multirow{2}{*}{72 hours after admission } & Yes & $8.33 \pm 4.05$ & \multirow{2}{*}{0.007} \\
\hline & & No & $6.5 \pm 3.67$ & \\
\hline & \multirow{2}{*}{ Last day of admission } & Yes & $8.74 \pm 4.45$ & \multirow{2}{*}{0.01} \\
\hline & & No & $7.0 \pm 4.7$ & \\
\hline \multirow{6}{*}{ High blood pressure } & \multirow{2}{*}{ At the time of admission } & Yes & $8.04 \pm 3.77$ & \multirow{2}{*}{0.26} \\
\hline & & No & $7.33 \pm 3.47$ & \\
\hline & \multirow{2}{*}{72 hours after admission } & Yes & $8.73 \pm 3.81$ & \multirow{2}{*}{0.10} \\
\hline & & No & $7.48 \pm 4.14$ & \\
\hline & \multirow{2}{*}{ Last day of admission } & Yes & $8.9 \pm 4.27$ & \multirow{2}{*}{0.09} \\
\hline & & No & $7.91 \pm 4.73$ & \\
\hline \multirow{6}{*}{ Diabetes } & \multirow{2}{*}{ At the time of admission } & Yes & $7.8 \pm 3.42$ & \\
\hline & & No & $7.54 \pm 3.7$ & 0.54 \\
\hline & 70 b fo d. & Yes & $7.89 \pm 3.63$ & $0=0$ \\
\hline & 72 hours after admission & No & $7.8 \pm 4.2$ & 0.52 \\
\hline & I act dov of admicsion & Yes & $8.29 \pm 3.8$ & 040 \\
\hline & Last day of admission & No & $8.33 \pm 4.91$ & 0.49 \\
\hline & H t b f & Yes & $8.02 \pm 3.48$ & 027 \\
\hline & At the time of admission & No & $7.47 \pm 3.65$ & 0.21 \\
\hline Heart dicease & 72 hours after a dmiccion & Yes & $9.12 \pm 4.12$ & 001 \\
\hline Heart disease & $T 2$ hours atter admission & No & $7.36 \pm 3.9$ & 0.01 \\
\hline & J ent dovef dmiccion & Yes & $9.45 \pm 4.81$ & \\
\hline & Last day of admission & No & $7.87 \pm 4.39$ & 0.05 \\
\hline & At the time of admicsion & Yes & $7.36 \pm 4.06$ & \\
\hline & At the time of admission & No & $7.67 \pm 3.53$ & 0.49 \\
\hline & & Yes & $8.05 \pm 5.18$ & \\
\hline Respiratory & 72 hours after admission & No & $7.79 \pm 3.83$ & 0.83 \\
\hline & I act dav of admiccion & Yes & $8.0 \pm 4.54$ & \\
\hline & Last day of admission & No & $8.37 \pm 4.57$ & 0.68 \\
\hline & & Yes & $11.0 \pm 3.96$ & \\
\hline & At the time of admission & No & $7.26 \pm 3.38$ & 0.001 \\
\hline & & Yes & $10.84 \pm 4.01$ & \\
\hline Kıdney disease & $T 2$ hours atter admission & No & $7.47 \pm 3.89$ & 0.005 \\
\hline & I oct dov of odmicsion & Yes & $3.4 \pm 13.11$ & 001 \\
\hline & Last day of admission & No & $8.02 \pm 4.49$ & 0.01 \\
\hline & s 1. & Yes & $14.00 \pm 1.73$ & 001 \\
\hline & At the time of admission & No & $7.50 \pm 3.52$ & 0.01 \\
\hline I iver diceose & 72 hours after admiccion & Yes & $14.00 \pm 7.07$ & 010 \\
\hline Liver disease & 72 hours after admission & No & $7.72 \pm 3.92$ & 0.10 \\
\hline & I & Yes & $17.66 \pm 1.15$ & 0004 \\
\hline & Last day of admission & No & $8.13 \pm 4.4$ & 0.004 \\
\hline & At the time of admicsion & Yes & $7.81 \pm 2.89$ & 061 \\
\hline & & No & $7.61 \pm 3.66$ & 0.01 \\
\hline & & Yes & $6.8 \pm 4.26$ & \\
\hline Neurologic disease & 72 hours after admission & No & $7.92 \pm 4.01$ & 0.37 \\
\hline & I ont do of olming & Yes & $7.54 \pm 4.82$ & \\
\hline & Last day of admission & No & $8.38 \pm 4.55$ & 0.65 \\
\hline & At the time of dmiccion & Yes & $8.0 \pm 3.78$ & 060 \\
\hline & At the time of admission & No & $7.59 \pm 3.6$ & 0.62 \\
\hline Melianonou & 72 boume oftar odmicrion & Yes & $8.66 \pm 4.79$ & 0,15 \\
\hline Malignancy & 72 hours after admission & No & $7.74 \pm 3.94$ & 0.45 \\
\hline & I act doy of admicsion & Yes & $9.35 \pm 5.25$ & 037 \\
\hline & Last day of admission & No & $8.22 \pm 4.49$ & 0.37 \\
\hline & At th tim of & Yes & $6.4 \pm 2.5$ & \\
\hline & At the time of admission & No & $7.67 \pm 3.63$ & 0.55 \\
\hline Rheumatic disense & 72 hours after admiccion & Yes & $5.66 \pm 1.15$ & 041 \\
\hline Rheumatic disease & $T 2$ hours after admission & No & $7.88 \pm 4.05$ & 0.41 \\
\hline & & Yes & $5.2 \pm 3.76$ & 007 \\
\hline & Last day of admission & No & $8.42 \pm 4.55$ & 0.07 \\
\hline
\end{tabular}

SOFA levels in patients with underlying disease were higher in all stages than in patients without underlying disease. Studies have shown that patients with a history of previous underlying disease are at 
higher risk of death [27]. Among the underlying diseases, liver disease was one of the cases that showed a significant relationship with SOFA levels at the beginning of hospitalization and also on the last day of hospitalization. Patients with COVID-19 who had a history of liver disease had a higher SOFA score than patients without liver disease at the time of admission. Patients with liver disease, especially uncompensated cirrhosis due to immune dysfunction, may be more susceptible to infection with SARS-COV-2 [28]. However, due to primary reports, 2-11\% of COVID-19 patients had a history of liver disease, but the true prevalence of COVID-19 among liver disease remains unknown [28, 29]. These patients show poor prognosis when admitted to the ICU, especially in the case of dysfunction or multiple organ failure [30]. Among the underlying diseases, kidney disease was one of the cases that had a significant relationship with SOFA levels at the beginning of hospitalization, 72 hours after hospitalization, and the last day of hospitalization in our survey. Patients with COVID-19 with a history of chronic kidney disease had higher SOFA scores than patients without kidney disease at all stages of the measurement. Having chronic underlying kidney disease is associated with a higher rate of in-hospital mortality. Also, patients with dialysis-dependent renal failure have the highest risk of in-hospital death compared to patients who do not undergo dialysis [31]. A study by Goswami et al. was conducted to evaluate the effectiveness of SOFA and other scoring systems on the outcome of patients with chronic kidney disease admitted to the ICU, which has represented that each SOFA score increase mortality significantly in 30-day period. Also, the sensitivity and specificity of SOFA $\geq 10.5$ in predicting 30-day mortality are $85 \%$ and $100 \%$, respectively [32]. Heart disease was another underlying disease in patients with COVID-19 that had a statistically significant relationship with SOFA levels in 72 hours after hospitalization and the last day of hospitalization. According to new studies, the SOFA score is a strong predictor in the cardiac intensive care unit [33]. Studies have shown that the clinical outcome is severe in patients with COVID-19 with a history of cardiovascular disease. A history of cardiovascular disease and the presence of cardiovascular risk factors make patients with COVID-19 vulnerable. In addition, COVID-19 can exacerbate underlying cardiovascular disease and even predispose these patients to new cardiac complications [34, 35]. Although several studies have been performed to evaluate the efficacy of the SOFA scoring system in patients with COVID-19 disease, few studies have systematically evaluated the accuracy of SOFA in diagnosing the severity of COVID-19 disease and its predictive value. Also, according to our investigation, this is the first study that evaluated the sofa score in 3 time periods in critically ill patients with COVID-19, which is the strength of our study. However, this study had some limitations; one of these limitations was the study's sample size. Certainly, studies with a large number of critically ill patients with COVID-19 can more accurately assess the efficiency of the SOFA scoring system. This study was a single-center study. Due to limited treatment resources and the impossibility of providing an intensive care bed for all critically ill patients, many of these patients were not admitted to the ICU. Extra multicenter studies should be done in longer time periods to enhance the SOFA Score's efficiency. Also, other scoring systems are widely used in the ICU. It is recommended that the effectiveness of these scoring systems in patients with COVID-19 be evaluated in future studies.

In summary, the SOFA scoring system at the beginning of hospitalization and 72 hours after hospitalization can be used to predict mortality in critically ill patients with COVID-19 who have been admitted to the ICU.

\section{Acknowledgments}

We would like to thank all individuals who volunteered to participate in this study. Furthermore, thank you to Razi hospital staff and specialists for assistance with conforming and recording cases.

\section{Author contributions}

Concept and Study design: MH, HKH, TY. Methods and experimental work: SR, ZAR, ST. Results analysis and conclusions: PR, MH, HKH, NF. Manuscript preparation: MH, ST, NF, ZAR. All authors read and approved the final version of manuscript.

\section{Conflict of interests}

No potential conflict of interest was reported by the authors.

\section{Ethical declarations}


All subjects gave their informed consent to participate in the study, and the study design was approved by the ethical committee of Guilan University of Medical Sciences by the code of ethics number IR.GUMS.REC.1399.509.

\section{Financial Support}

Self-funding.

\section{References}

1. Chen N, Zhou M, Dong X, Qu J, Gong F, Han Y, et al. Epidemiological and clinical characteristics of 99 cases of 2019 novel coronavirus pneumonia in Wuhan, China: a descriptive study. Lancet. 2020; 395(10223):507-13.

2. Guo YR, Cao QD, Hong ZS, Tan YY, Chen SD, Jin HJ, et al. The origin, transmission and clinical therapies on coronavirus disease 2019 (COVID-19) outbreak - an update on the status. Mil Med Res. 2020; $7(1): 11$.

3. Abdelrahman Z, Li M, Wang X. Comparative Review of SARSCoV-2, SARS-CoV, MERS-CoV, and Influenza A Respiratory Viruses. Front Immunol. 2020; 11:552909.

4. Han H, Yang L, Liu R, Liu F, Wu KL, Li J, et al. Prominent changes in blood coagulation of patients with SARS-CoV-2 infection. Clin Chem Lab Med. 2020; 58(7):1116-20.

5. Yang X, Yu Y, Xu J, Shu H, Xia J, Liu H, et al. Clinical course and outcomes of critically ill patients with SARS-CoV-2 pneumonia in Wuhan, China: a single-centered, retrospective, observational study. Lancet Respir Med. 2020; 8(5):475-81.

6. Bhatraju PK, Ghassemieh BJ, Nichols M, Kim R, Jerome KR, Nalla AK, et al. Covid-19 in Critically Ill Patients in the Seattle Region - Case Series. N Engl J Med. 2020; 382(21):2012-22.

7. Chen Q, Xu L, Dai Y, Ling Y, Mao J, Qian J, et al. Cardiovascular manifestations in severe and critical patients with COVID-19. Clin Cardiol. 2020; 43(7):796-802.

8. Choudry FA, Hamshere SM, Rathod KS, Akhtar MM, Archbold RA, Guttmann OP, et al. High Thrombus Burden in Patients With COVID-19 Presenting With ST-Segment Elevation Myocardial Infarction. J Am Coll Cardiol. 2020; 76(10):1168-76.

9. Liu Z, Meng Z, Li Y, Zhao J, Wu S, Gou S, et al. Prognostic accuracy of the serum lactate level, the SOFA score and the qSOFA score for mortality among adults with Sepsis. Scand J Trauma Resusc Emerg Med. 2019; 27(1):51.

10. Raith EP, Udy AA, Bailey M, McGloughlin S, MacIsaac C, Bellomo R, et al. Prognostic Accuracy of the SOFA Score, SIRS Criteria, and qSOFAScore for In-Hospital Mortality Among Adults With Suspected Infection Admitted to the Intensive Care Unit. Jama. 2017; 317(3):290-300.

11. Khwannimit B, Bhurayanontachai R, Vattanavanit V. Comparison of the accuracy of three early warning scores with SOFA score for predicting mortality in adult sepsis and septic shock patients admitted to intensive care unit. Heart Lung. 2019; 48(3):240-4.

12. Chen T, Wu D, Chen H, Yan W, Yang D, Chen G, et al. Clinical characteristics of 113 deceased patients with coronavirus disease 2019: retrospective study. Bmj. 2020; 368:m1091.

13. Vincent JL, Moreno R, Takala J, Willatts S, De Mendonça A, Bruining $\mathrm{H}$, et al. The SOFA (Sepsis-related Organ Failure
Assessment) score to describe organ dysfunction/failure. On behalf of the Working Group on Sepsis-Related Problems of the European Society of Intensive Care Medicine. Intensive Care Med. 1996; 22(7):707-10.

14. Schoe A, Bakhshi-Raiez F, de Keizer N, van Dissel JT, de Jonge E. Mortality prediction by SOFA score in ICU-patients after cardiac surgery; comparison with traditional prognostic-models. BMC Anesthesiol. 2020; 20(1):65.

15. Singer M, Deutschman CS, Seymour CW, Shankar-Hari M, Annane D, Bauer M, et al. The Third International Consensus Definitions for Sepsis and Septic Shock (Sepsis-3). Jama. 2016; 315(8):801-10.

16. Trancă S, Petrișor C, Hagău N, Ciuce C. Can APACHE II, SOFA, ISS, and RTS Severity Scores be used to Predict Septic Complications in Multiple Trauma Patients? J Crit Care Med (Targu Mures). 2016; 2(3):124-30.

17. Yang Z, Hu Q, Huang F, Xiong S, Sun Y. The prognostic value of the SOFA score in patients with COVID-19: A retrospective, observational study. Medicine (Baltimore). 2021; 100(32):e26900.

18. Ji Y, Ma Z, Peppelenbosch MP, Pan Q. Potential association between COVID-19 mortality and health-care resource availability. Lancet Glob Health. 2020; 8(4):e480.

19. Antommaria AHM, Gibb TS, McGuire AL, Wolpe PR, Wynia MK, Applewhite MK, et al. Ventilator Triage Policies During the COVID-19 Pandemic at U.S. Hospitals Associated With Members of the Association of Bioethics Program Directors. Ann Intern Med. 2020; 173(3):188-94.

20. Swiss Academy Of Medical S. COVID-19 pandemic: triage for intensive-care treatment under resource scarcity. Swiss Med Wkly. 2020; 150:w20229.

21. Lambden S, Laterre PF, Levy MM, Francois B. The SOFA scoredevelopment, utility and challenges of accurate assessment in clinical trials. Crit Care. 2019; 23(1):374.

22. Grissom CK, Brown SM, Kuttler KG, Boltax JP, Jones J, Jephson AR, et al. A modified sequential organ failure assessment score for critical care triage. Disaster Med Public Health Prep. 2010; 4(4):277-84.

23. Ferreira FL, Bota DP, Bross A, Mélot C, Vincent JL. Serial evaluation of the SOFA score to predict outcome in critically ill patients. Jama. 2001; 286(14):1754-8.

24. Liu S, Yao N, Qiu Y, He C. Predictive performance of SOFA and qSOFA for in-hospital mortality in severe novel coronavirus disease. Am J Emerg Med. 2020; 38(10):2074-80.

25. Raschke RA, Agarwal S, Rangan P, Heise CW, Curry SC. Discriminant Accuracy of the SOFA Score for Determining the Probable Mortality of Patients With COVID-19 Pneumonia Requiring Mechanical Ventilation. Jama. 2021; 325(14):1469-70. 26. Gupta S, Hayek SS, Wang W, Chan L, Mathews KS, Melamed ML, et al. Factors Associated With Death in Critically Ill Patients With Coronavirus Disease 2019 in the US. JAMA Intern Med. 2020; 180(11):1436-47.

27. Peng YD, Meng K, Guan HQ, Leng L, Zhu RR, Wang BY, et al. [Clinical characteristics and outcomes of 112 cardiovascular disease patients infected by 2019-nCoV]. Zhonghua Xin Xue Guan Bing Za Zhi. 2020; 48(6):450-5.

28. Zhou F, Yu T, Du R, Fan G, Liu Y, Liu Z, et al. Clinical course and risk factors for mortality of adult inpatients with COVID-19 in 


\section{Haghighi et al.}

Wuhan, China: a retrospective cohort study. Lancet. 2020; 395(10229):1054-62.

29. Rosenblatt R, Verna EC. COVID 19: Management of Decompensated Cirrhosis and Liver Transplant Recipients. Clin Liver Dis (Hoboken). 2020; 15(5):200-3.

30. Pan HC, Jenq CC, Tsai MH, Fan PC, Chang CH, Chang MY, et al. Scoring systems for 6-month mortality in critically ill cirrhotic patients: a prospective analysis of chronic liver failure - sequential organ failure assessment score (CLIF-SOFA). Aliment Pharmacol Ther. 2014; 40(9):1056-65.

31. Flythe JE, Assimon MM, Tugman MJ, Chang EH, Gupta S, Shah J, et al. Characteristics and Outcomes of Individuals With Pre-existing Kidney Disease and COVID-19 Admitted to Intensive Care Units in the United States. Am J Kidney Dis. 2021; 77(2):190203.e1.

32. Goswami J, Balwani MR, Kute V, Gumber M, Patel M, Godhani U. Scoring systems and outcome of chronic kidney disease patients admitted in intensive care units. Saudi J Kidney Dis Transpl. 2018; 29(2):310-7.

33. Elias A, Agbarieh R, Saliba W, Khoury J, Bahouth F, Nashashibi J, et al. SOFA score and short-term mortality in acute decompensated heart failure. Sci Rep. 2020; 10(1):20802.

34. Huang C, Wang Y, Li X, Ren L, Zhao J, Hu Y, et al. Clinical features of patients infected with 2019 novel coronavirus in Wuhan, China. Lancet. 2020; 395(10223):497-506.

35. Matsushita K, Ding N, Kou M, Hu X, Chen M, Gao Y, et al. The Relationship of COVID-19 Severity with Cardiovascular Disease and Its Traditional Risk Factors: A Systematic Review and MetaAnalysis. Glob Heart. 2020; 15(1):64. 